\title{
DYNAMICAL SYSTEMS TECHNIQUES IN COSMOLOGY. AN EXAMPLE: LQC AND THE EINSTEIN STATIC UNIVERSE
}

\author{
LUCA PARISI
}

Communicated by Akira Yoshioka

Abstract. Dynamical Systems theory is a valuable tool in the profound study of Cosmology, as qualitative methods allow to characterize cosmological solutions on the basis of their relevant physical features (e.g. stability and asymptotic behaviour). Here we briefly review some well established results for cosmological models in the framework of General Relativity. Then we consider a family of modified cosmological models which are gaining more and more relevance, namely Loop Quantum Cosmologies. In particular we analyze the geometrical structure and dynamical properties of the model presented in earlier.

\section{Introduction}

We consider the application of dynamical systems theory to mathematical models from classical and semiclassical Cosmology. In particular, the Einstein Static (ES) universe in General Relativity (GR) is reviewed along with its stability properties. Then static solutions of the so called semiclassical Loop Quantum Cosmology (LQC) modified equations for homogeneous and isotropic closed cosmological models $(\mathrm{K}=1)$ with a cosmological constant $\Lambda$ are considered.

The paper is structured as follows. In the first section we review some basic notions about Cosmology which will be helpful for the following discussion. In the second section we introduce some standard definitions from Dynamical Systems theory which are employed, together with numerical integrations, in the following sections. In the third section, we describe the ES model in GR and the dynamical system approach shows that the ES solution is unstable to homogeneous perturbations. Then we focus on Loop Quantum Gravity [17] techniques that, applied to Cosmology, give rise to LQC [4]. LQC strongly modifies the high-energy dynamics of Friedman-Robertson-Walker models. One of its more remarkable features is that it removes the big-bang singularity [15]. We show that LQC modifications can lead to an ES model which is neutrally stable for a large enough positive value of $\Lambda$. 


\section{Basics of Cosmology}

Many experimental evidences show that the universe (as far as we can observe it) is fairly homogeneous and isotropic on large enough scales (i.e., $150 \mathrm{~h}^{-1} \mathrm{Mpc}$ where $1 M p c \simeq 3 \times 10^{22} \mathrm{~m}$ and $h \simeq 0.7$ is a parameter related to the expansion rate of the universe). It is quite natural to describe its evolution as a whole in the framework of GR assuming homogeneity and isotropy as a basic principle in nature (the Cosmological Principle). In this picture one neglects the small perturbations in the energy density and concerns only with the evolution of the background. The Friedmann-Lemaitre models are based on the assumption that the universe is isotropic around every point, which necessarily implies homogeneity. The resulting Robertson-Walker metric has the form

$$
\mathrm{d} s^{2}=-\mathrm{d} t^{2}+a(t)\left[\mathrm{d} \chi^{2}+S_{k}^{2}(\chi)\left(\mathrm{d} \theta^{2}+\sin ^{2}(\theta) \mathrm{d} \varphi^{2}\right)\right]
$$

with $S_{k}^{2}(\chi)=(\sin (\chi), \chi, \sinh (\chi))$ for the three values of the label $K=(1,0,-1)$. $K$ describes the curvature of the spatial sections. $K=+1$ corresponds to positively curved spatial sections (locally isometric to three-spheres); $K=0$ corresponds to local flatness, and $K=-1$ corresponds to negatively curved (locally hyperbolic) spatial sections.

The matter content of the model is described by a stress-energy tensor which is forced by the symmetry of the model to have the algebraic form of a perfect fluid:

$$
T_{\mu \nu}=(\rho+p) U^{\mu} U_{\nu}+p g_{\mu \nu}
$$

where $U^{\mu}$ is the fluid four-velocity; $\rho$ and $p$ are energy density and pressure in the rest frame of the fluid respectively.

Plugging the metric from equation (1) into the Einstein equations one gets two differential equations governing the evolution of the scale factor $a(t)$. They are the Raychaudhuri equation

$$
\dot{H}=-\frac{\kappa}{2} \rho(1+w)+\frac{K}{a^{2}}
$$

and the Friedmann equation

$$
H^{2}=\frac{\kappa}{3} \rho+\frac{\Lambda}{3}-\frac{K}{a^{2}}
$$

where $H=\dot{a} / a$ is the Hubble parameter, $\kappa=8 \pi G / 3$ and the dot denotes derivative with respect to cosmic time $t$. Moreover, energy conservation is expressed in 
GR by the vanishing of the covariant divergence of the energy-momentum tensor, $\nabla_{\mu} T^{\mu \nu}=0$ yielding a single energy-conservation equation

$$
\dot{\rho}+3 H(\rho+p)=0 .
$$

These three equations are not independent, indeed the Friedmann equation is a first integral of equation (3) and equation (5) whenever $\dot{a} \neq 0$ so that it is a constraint which relates the expansion rate of the universe to the energy density the spatial curvature of the universe.

The model can be easily generalized to the case of several different energy density and pressure components $\rho_{i}$ and $p_{i}$. Adding a cosmological constant to the Einstein's equation is equivalent to including a new component of the energy density in the universe described by an energy-momentum tensor of the form $T_{\mu \nu}=-\frac{\Lambda}{8 \pi G} g_{\mu \nu}$ with pressure and density $\rho_{\Lambda}=\frac{\Lambda}{8 \pi G}$ and $p_{\Lambda}=-\rho_{\Lambda}$.

It can be shown that, under very general condition (i.e., dominant energy condition) holding for all the known kinds of ordinary matter, the system of equation (3), equation (4) and equation (5) lead to a singularity. Roughly speaking, this means that the system describes a pathological behaviour (e.g. the curvature blows-up, the energy density diverges) [19]. This is one of the main motivations to look for a quantum theory of gravitation free such inconsistencies.

\section{A Primer on Dynamical Systems}

In this section a very short introduction to Dynamical Systems is provided. This section is aimed to account the reader with some basic definition and theorems employed in the following sections.

It is customary to recast the evolution equations of Cosmology as an autonomous dynamical system in order to characterize relevant features (e.g. the late time behaviour of the universe, the attractor solutions). A more detailed introduction to dynamical systems is provided in [1] and applications of Dynamical System theory to classical cosmology are discussed in [18].

Let $\mathbf{X}$ be a metric space.

Definition 1. A (continuous) dynamical system is a one-parameter family of invertible maps $\phi_{t}: \mathbf{X} \rightarrow \mathbf{X}, t \in \mathbb{R}$, such that

- $\phi_{0}=\mathrm{Id}$

- $\phi_{t_{1}+t_{1}}=\phi_{t_{1}}+\phi_{t_{2}}, \quad$ for all $t_{1}, t_{2} \in \mathbb{R}$ 
- $\phi_{-t}=\left(\phi_{t}\right)^{-1}, \quad t \in \mathbb{R}$.

Definition 2. A Dynamical System which does not depend explicitly on time is said to be autonomous.

Dynamical System of practical interest are generally in the form of vector field on a state space $\mathbf{X}$

$$
\dot{x}=f(x) .
$$

The sate space or phace space $\mathbf{X}$ can be a differential manifold (e.g. a sphere, a torus etc.). More simply one can have a vector space $\mathbf{X}=\mathbb{R}^{n}, x=\left(x_{1}, \ldots, x_{n}\right)$, in which case equation (6) represents a system of ordinary differential equations. Then the one parameter maps considered below are naturally interpreted as the flow of equation (6).

Definition 3. An equilibrium solution or fixed point or critical point is a point $\bar{x} \in \mathbf{X}$ such that $f(\bar{x})=0$.

One of the main goal of Dynamical Systems theory is to determine the future asymptotic behaviour (i.e., $t \rightarrow \infty$ ), because one is interested in the long-term evolution of the corresponding physical system. In cosmology one is also interested in the past asymptotic behaviour (near the initial singularity). Two important definition can be given.

Definition 4. A solution $\bar{x}(t)$ of a dynamical system is said to be (Liapunov) stable if, given $\varepsilon>0$, there exist a $\delta=\delta(\varepsilon)$ such that, for any other solution, $y(t)$, satisfying $\left|\bar{x}\left(t_{0}\right)-y\left(t_{0}\right)\right|<\delta$, then $|\bar{x}(t)-y(t)|<\varepsilon$ for $t>t_{0}, t_{0} \in \mathbb{R}$. $A$ solution which is not stable is said to be unstable.

Definition 5. A solution $\bar{x}(t)$ of a dynamical system is said to be asymptotically stable if it is Liapunov stable and if there exist a constant $b>0$ such that, if $\left|\bar{x}\left(t_{0}\right)-y\left(t_{0}\right)\right|<b$ then $\lim _{t \rightarrow \infty}|\bar{x}(t)-y(t)|=0$.

These definitions do not actually provide us with a method for determining whether or not a given solution is stable. Qualitative information can be gained by studying the local properties of the flow in the neighborhood of the equilibrium points.

Thus let's consider a linear differential equation $\dot{x}=A x$ on $\mathbb{R}^{n}$, where $A$ is an $n \times n$ matrix of real numbers, three subspaces of $\mathbb{R}^{n}$ are defined:

the stable subspace

the unstable subspace

the centre subspace

$$
\begin{aligned}
& E^{s}=\operatorname{span}\left(s_{1}, \ldots, s_{n_{s}}\right) \\
& E^{u}=\operatorname{span}\left(u_{1}, \ldots, u_{n_{n}}\right) \\
& E^{c}=\operatorname{span}\left(c_{1}, \ldots, c_{n_{c}}\right)
\end{aligned}
$$


where $s_{1}, \ldots, s_{n_{s}}$ are the generalized eigenvectors of $A$ whose eigenvalues have negative real parts, $u_{1}, \ldots, u_{n_{u}}$ are those whose eigenvalues have positive real parts and $c_{1}, \ldots, c_{n_{c}}$ are those whose eigenvalues have zero real parts. Clearly $E^{s} \oplus E^{u} \oplus$ $E^{c}=\mathbb{R}^{n}$ and

$$
\begin{array}{ll}
x \in E^{s} & \Rightarrow \quad \lim _{t \rightarrow+\infty} \exp (A t) x=0 \\
x \in E^{u} & \Rightarrow \quad \lim _{t \rightarrow-\infty} \exp (A t) x=0 .
\end{array}
$$

This is a description of the asymptotic behaviour of the linear system: all initial states in the stable subspace are attracted to the equilibrium point the 0 vector, while all initial states in the unstable subspace are repelled by 0.

Let us turn to nonlinear systems represented by equation (6). Once that a differential equation have been linearized around any of its equilibrium points and their stability have been determined, the behaviour of the resulting linear system is related to the original non-linear system by the Hartman-Grobman theorem. The linearization of equation (6) at an equilibrium point $\bar{x}$ is given by

$$
\dot{x}=\operatorname{Df}(\bar{x})(x-\bar{x})
$$

where $D$ is the derivative of $f$. When an equilibrium point $\bar{x}$ is hyperbolic, that is, when all the eigenvalues of $D f(\bar{x})$ have non-vanishing real part, the HartmanGrobman theorem ensures that in a neighborhood of $\bar{x}$ exists a homomorphism which maps orbits of the flow generated by the original nonlinear differential equation onto orbits of the corresponding linearized system preserving their orientation (those orbits are said topologically equivalent). Thus the stability properties of a nonlinear dynamical system near a hyperbolic fixed point are qualitatively described by its linearization at that point.

\section{The Einstein Static Universe in GR and Loop Quantum Cosmology}

The ES universe in GR is a closed Friedman-Robertson-Walker (FRW) model with a perfect fluid and a cosmological constant. It is well known that Einstein himself was not comfortable with the idea of an expanding or collapsing universe, thus he introduced a parameter, nowadays called cosmological constant, to balance the effect of gravity obtaining a static solution (for a detailed description of ES universe see [11]). Later on it was proved that the ES solution is unstable to homogeneous perturbations [7]. More recently it was shown that the ES universe is neutrally stable to inhomogeneous scalar perturbations with high enough sound speed and to vector and tensor perturbations [2]. 
The stability of ES models in high-energy modifications of GR is an interesting mathematical question, and is also relevant for scenarios in which the ES is an initial state for a past-eternal inflationary cosmology, the so-called Emergent universe scenario [9]. The standard model of cosmology (see e.g. [16]) has a flat infinite spatial geometry, and experiences a period of primordial inflationary expansion, which is preceded by a big bang singularity in the classical theory. Observations however do not prove that the geometry is flat: the universe could have nonzero spatial curvature, as long as the late-time effect of this curvature is very small. In particular, a positive curvature allows for an "Emergent universe" that originates asymptotically in the past as an Einstein static universe, and then inflates and later reheats to a hot big bang era. This model generalizes the Eddington-Lemaitre model [8], and is a counter-example to the notion that inflation can never be past-eternal and thus cannot avoid an initial singularity because it is closed, it avoids the theorems showing that inflation cannot be past eternal [5].

Generalizations of the Einstein static solution in high-energy modifications to GR have been considered in the Randall-Sundrum braneworld scenario [10] and in $f(R)$ theories [6]. The stability of ES models against homogeneous perturbations has also been investigated in [3] for the case of $R+\alpha R^{2}$ gravity. Eventually, in it was proved that Einstein static universes are always unstable to either homogeneous or inhomogeneous perturbations in the context of generic $f(R)$ models [14].

Another theory leading to high-energy modifications of GR is Loop Quantum Cosmology (LQC) [4], which is a canonical quantization of homogeneous cosmological spacetimes based on Loop Quantum Gravity [17]. The gravitational phase-space variables are an $\mathfrak{s u}(2)$ valued connection and conjugate triad, and the elementary variables underlying the quantization are the holonomies of the connection and the fluxes of the triad. The quantum theory obtained from LQC turns out to be inequivalent to Wheeler-de Witt quantization (the LQC polymer representation is different from the usual Wheeler-de Witt Schrödinger representation). Wheeler-de Witt quantization does not resolve the cosmological singularity, but in LQC a generic resolution of such singularities has been obtained (see [15] and references therein).

Mulryne et al. [12] used the scalar-field modification approach to investigate the stability of the Einstein static model to homogeneous perturbations. They found that the new LQC Einstein static model is a centre fixed point in phase space, i.e., a neutrally stable point, for a massless scalar field with $w \equiv p_{\phi} / \rho_{\phi}=1$. This modification of stability behaviour has important consequences for the Emergent 
universe scenario, since it ameliorates the fine-tuning that arises from the fact that the Einstein static is an unstable saddle in GR.

Here we consider the same question, but using the LQC gravitational modifications, which typically become important at lower energy scales than the modifications to the scalar field dynamics, thus neglecting the higher energy modifications to matter. We consider a perfect fluid with $p=w \rho$ and $w>-1$. We show that, for all $w>-1 / 3$, there is a new centre, i.e., a neutrally stable point representing an Einstein static model, but only if the cosmological constant $\Lambda$ is above a critical scale: $\Lambda>6.6 \pi M_{P}^{2}$.

\subsection{A Characterization of the Einstein Static Universe in GR through Dynamical System}

Let us consider the system of equations (3), (4) and (5). After simple manipulations it can be explicitly written in the form of a planar (that is, two-dimensional) autonomous dynamical system in the variables $H$ and $\rho$.

$$
\begin{aligned}
\dot{H} & =-H^{2}-\frac{\kappa}{6} \rho(1+3 w)+\frac{\Lambda}{3} \\
\dot{\rho} & =-3 H(\rho+p) .
\end{aligned}
$$

Among the other fixed points the system admits a critical point which is a static solution $\dot{a}=\dot{H}=\dot{\rho}=0$. It is the Einstein static universe defined by the conditions

$$
\rho_{G R}=\frac{2 \Lambda}{\kappa(1+3 w)}, \quad a_{G R}^{2}=\frac{2}{\kappa \rho_{G R}(1+w)} .
$$

Assuming a positive cosmological constant $\Lambda>0$, from the conditions $a^{2}>0$ and $\rho>0$ it follows that the solution exists when $w>-1 / 3$.

The eigenvalues of the linearized system are

$$
\lambda_{G R}= \pm \sqrt{\Lambda(1+w)} .
$$

These are real with opposite signs for $\Lambda>0$ and $w>-1 / 3$, so that the fixed point is unstable (of the saddle type). Thus the classic ES solution in GR is unstable to homogeneous perturbations. A deeper analysis of the ES solution in GR in comparison with new static solutions is provided in the following.

\subsection{Critical Points of Closed FRW Models in LQC}

Let us now turn to the semiclassical LQC models. The loop quantum effects that we investigate manifest themselves in the form of a modification to the classical 
Friedmann equation (4). For the closed FRW model, the explicit form is given by

$$
H^{2}=\left(\frac{\kappa}{3} \rho+\frac{\Lambda}{3}-\frac{1}{a^{2}}\right)\left(1-\frac{\rho}{\rho_{c}}-\frac{\Lambda}{\kappa \rho_{c}}+\frac{3}{\kappa \rho_{c} a^{2}}\right)
$$

where $\kappa=8 \pi G=8 \pi / M_{P}^{2}$, and the critical LQC energy density is

$$
\rho_{c} \approx 0.82 M_{P}^{4}
$$

It is evident that the first term in parentheses is the classical right hand side of the Friedmann equation, with the quantum modifications appearing in the second term. The classical GR limit is achieved in the limit as $\rho_{c}$ goes to infinity whence the second term approaches unity. The classical energy conservation equation continues to hold ${ }^{1}$

$$
\dot{\rho}=-3 H \rho(1+w) .
$$

Note that $H^{2} \geq 0$ imposes the limits

$$
\frac{3}{a^{2}} \leq \kappa \rho+\Lambda \leq \kappa \rho_{c}+\frac{3}{a^{2}} .
$$

The modified Raychaudhuri equation follows from equations (14) and (12):

$$
\begin{aligned}
\dot{H}= & -\frac{\kappa}{2} \rho(1+w)\left(1-\frac{2 \rho}{\rho_{c}}-\frac{2 \Lambda}{\kappa \rho_{c}}\right) \\
& +\left[1-\frac{2 \rho}{\rho_{c}}-\frac{2 \Lambda}{\kappa \rho_{c}}-\frac{3 \rho(1+w)}{\rho_{c}}\right] \frac{1}{a^{2}}+\frac{6}{\kappa \rho_{c} a^{4}} .
\end{aligned}
$$

We will find the critical points to the system of equations (14) and (16) as well as

$$
\dot{a}=a H
$$

which follows from the definition of $H$. The solution space is a two-dimensional surface in the three-dimensional $(\rho, a, H)$ space, defined by the Friedman constraint (12). The system admits two critical points, which are static solutions $\dot{a}=\dot{H}=\dot{\rho}=0$. The first critical point is the standard GR Einstein static universe, while the second is a new LQC Einstein static universe

$$
\begin{array}{rlrl}
\rho_{G R} & =\frac{2 \Lambda}{\kappa(1+3 w)}, & a_{G R}^{2}=\frac{2}{\kappa \rho_{G R}(1+w)} \\
\rho_{L Q}=\frac{2\left(\Lambda-\kappa \rho_{c}\right)}{\kappa(1+3 w)}, & a_{L Q}^{2}=\frac{2}{\kappa \rho_{L Q}(1+w)} .
\end{array}
$$

\footnotetext{
${ }^{1}$ We are not considering the inverse volume effects that would modify the scalar field energy density and hence modify the energy conservation equation. For the closed model there are indications that the inverse volume effects are negligible if it is required that the universe reach macroscopic size.
} 
The conditions under which these static solutions exist are summarized in Table 1, and follow from $a^{2}>0$ and $\rho>0$.

Table 1. Conditions for the existence of Einstein static critical points.

\begin{tabular}{c|lc}
\hline GR & $\Lambda>0$ & $w>-1 / 3$ \\
& $\Lambda<0$ & $-1<w<-1 / 3$ \\
\hline LQ & $\Lambda<\kappa \rho_{c}$ & $-1<w<-1 / 3$ \\
& $\Lambda>\kappa \rho_{c}$ & $w>-1 / 3$ \\
\hline
\end{tabular}

A remarkable feature of the new LQ fixed point is that it is possible to have an Einstein static universe even for vanishing cosmological constant. Indeed, as one can see from equation (19) and Table 2, when $\Lambda=0$ the LQ fixed point exists and is unstable.

For the system of equations (14), (16) and (17), linearized stability analysis fails to give complete information about the properties of the two critical points, when they both exist, since in all cases, for one of the two points there is always a pair of complex eigenvalues with vanishing real part. In this case the linearization theorem (see e.g. [1]) tells us that a fixed point which is a centre for the linearized system is not necessarily a centre for the full nonlinear system. In addition, the linearization of equations (14), (16) and (17) always leads to a third vanishing eigenvalue, simply because the actual dynamics is two-dimensional because of the modified Friedman equation (12). Therefore, using this constraint, it is convenient to rewrite the system reducing the number of equations to two, the dimension of the configuration space. Solving equation (12) for $a^{2}$, we find that

$$
a^{2}=f_{ \pm}(\rho, H)
$$

where

$$
f_{ \pm}=\frac{3}{2} \frac{\left[2(\kappa \rho+\Lambda)+\kappa \rho_{c}\left(1 \pm \sqrt{1-12 H^{2} / \kappa \rho_{c}}\right)\right]}{(\kappa \rho+\Lambda)^{2}+\kappa \rho_{c}\left(3 H^{2}-\kappa \rho-\Lambda\right)} .
$$

Substituting this into equation (16), we find two branches for the time derivative of the Hubble parameter

$$
\begin{array}{llll}
\text { LQ }: & \dot{\rho}=-3 H \rho(1+w) & \text { and } & \dot{H}=F_{+}(\rho, H) \\
\text { GR }: \quad \dot{\rho}=-3 H \rho(1+w) & \text { and } & \dot{H}=F_{-}(\rho, H)
\end{array}
$$


where

$$
\begin{aligned}
F_{ \pm}= & -\frac{\kappa}{2}(1+w) \rho\left(1-\frac{2 \rho}{\rho_{c}}-\frac{2 \Lambda}{\kappa \rho_{c}}\right)+\frac{6}{\kappa \rho_{c} f_{ \pm}^{2}} \\
& +\frac{1}{f_{ \pm}}\left[1-\frac{2 \rho}{\rho_{c}}-\frac{2 \Lambda}{\kappa \rho_{c}}-3(1+w) \frac{\rho}{\rho_{c}}\right]
\end{aligned}
$$

In the classical limit

$$
\begin{aligned}
& \lim _{\rho_{c} \rightarrow \infty} f_{+}=0 \\
& \lim _{\rho_{c} \rightarrow \infty} f_{-}=\frac{3}{\kappa \rho-3 H^{2}+\Lambda}
\end{aligned}
$$

where the second equation is the GR Friedman equation. Thus the two branches in equation (20) recover the GR and the new quantum static solution respectively. In addition,

$$
\begin{aligned}
& \lim _{\rho_{c} \rightarrow \infty} F_{+}=\infty \\
& \lim _{\rho_{c} \rightarrow \infty} F_{-}=-H^{2}-\frac{\kappa \rho}{6}(1+w)+\frac{\Lambda}{3}
\end{aligned}
$$

where the first limit is consistent with equation (25), and the second limit gives the GR Raychaudhuri equation.

The system (22) admits the static solution with $\rho_{L Q}$ as in equation (19). Substituting this into equation (21) reproduces $a_{L Q}^{2}$ as in equation (19). We evaluate the eigenvalues of the Jacobian matrix at this point, to find the two eigenvalues

$$
\lambda_{L Q}= \pm \sqrt{\left(\kappa \rho_{c}-\Lambda\right)(1+w)} .
$$

Thus the LQ fixed point is either unstable (of the saddle kind), when $\kappa \rho_{c}>\Lambda$ and $-1<w<-1 / 3$, or a centre for the linearized system, i.e., a neutrally stable fixed point, when $\kappa \rho_{c}<\Lambda$ and $w>-1 / 3$. (The limits take into account the conditions in Table 1.) Again, for the latter point the linearized analysis is not sufficient and therefore we turn to a numerical analysis in the next section.

For the system equation (23), we find the GR static solution, and the eigenvalues of the linearized system are

$$
\lambda_{G R}= \pm \sqrt{\Lambda(1+w)} .
$$

These are real with opposite signs for $\Lambda>0$ and $w>-1 / 3$, so that the fixed point is unstable (of the saddle type). For $\Lambda<0$ and $-1<w<-1 / 3$, the fixed 
point is a centre, as confirmed by numerical analysis. It does not appear to be widely known that the GR Einstein static universe can be neutrally stable when $\Lambda<0$ (see also [3]).

The results of the linearized stability analysis are summarized in table below.

Table 2. Eigenvalues for the critical points in Table 1.

\begin{tabular}{|c|c|c|}
\hline & $\lambda_{1}$ & $\lambda_{2}$ \\
\hline \multicolumn{3}{|l|}{ GR } \\
\hline$\Lambda>0 \quad$ and $\quad w>-1 / 3$ & $>0$ & $<0$ \\
\hline$\Lambda<0 \quad$ and $\quad-1<w<-1 / 3$ & $\begin{array}{l}\operatorname{Re}\left(\lambda_{1}\right)=0 \\
\operatorname{Im}\left(\lambda_{1}\right)>0\end{array}$ & $\begin{array}{l}\operatorname{Re}\left(\lambda_{2}\right)=0 \\
\operatorname{Im}\left(\lambda_{2}\right)<0\end{array}$ \\
\hline \multicolumn{3}{|l|}{ LQ } \\
\hline and $\quad-1<w<-1 / 3$ & $>0$ & $<0$ \\
\hline$\Lambda>\kappa \rho_{c} \quad$ and $\quad w>-1 / 3$ & $\begin{array}{l}\operatorname{Re}\left(\lambda_{1}\right)=0 \\
\operatorname{Im}\left(\lambda_{1}\right)>0\end{array}$ & $\begin{array}{l}\operatorname{Re}\left(\lambda_{2}\right)=0 \\
\operatorname{Im}\left(\lambda_{2}\right)<0\end{array}$ \\
\hline
\end{tabular}

\subsection{Numerical Integration}

In order to extend the linearized stability analysis, we perform numerical integrations of the systems (22) and (23). We also integrate the nonlinear system of equations (14), (16) and (17), with initial conditions fulfilling the Friedman constraint (12), in order to show the full configuration space diagrams and gain a better understanding of the dynamics. Here we take $\Lambda>0$ and $w=1$ and we focus on the case $\Lambda>\kappa \rho_{c}$ (for more details about the case $\Lambda<\kappa \rho_{c}$ see [13]).

The $(H, \rho)$ plot in Fig. 1 is obtained by integrating the system (22) and (23) in some neighborhood of the fixed point. It shows that the LQ fixed point is a centre. More insight can be obtained by plotting the whole $3 \mathrm{D}$ space $(H, \rho, a)$ for a wide range of initial conditions, shown in Fig. 2. The trajectories lie on the two-dimensional Friedman constraint surface. The behaviour near the fixed point agrees with the linearized stability analysis, but new interesting features arise. For initial conditions far enough from the fixed point, there are trajectories that wrap around the Friedman tube, so that cyclic models are possible even if they are not related with the centre fixed point, since they cannot be shrunk to a point.

This behaviour can also be interpreted through a plot of the energy density against 


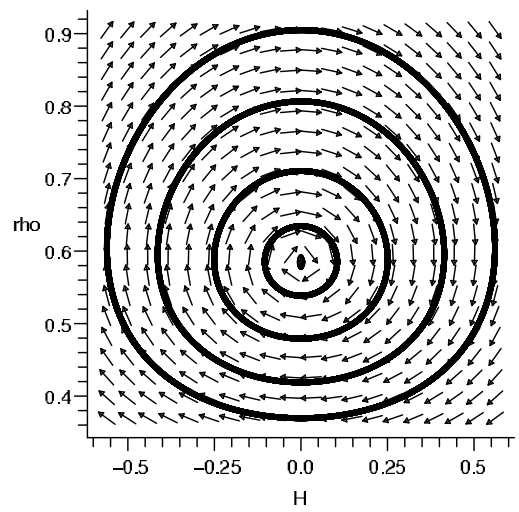

Figure 1. Dynamical behaviour of the system around the LQ fixed point for the case $\Lambda>\kappa \rho_{c}$, with $\Lambda / \kappa=2, w=1$ (using units $M_{P}=1$ ).

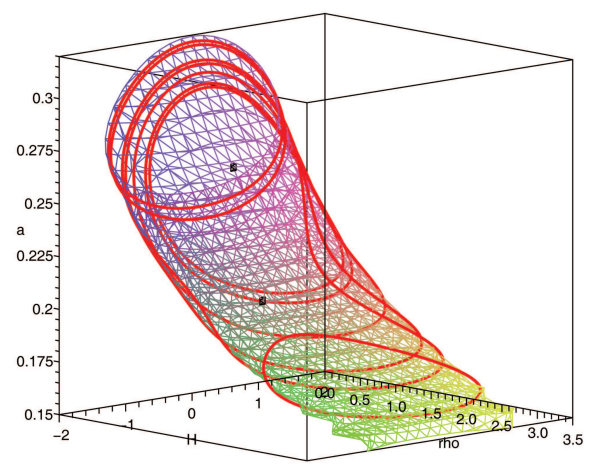

Figure 2. Trajectories on the Friedman constraint surface, for the same parameters as in Fig. 1. The GR fixed point is at the bottom of the Friedman surface, while the LQ point is at the top. Note that some trajectories wrap around the "tube" but cannot be shrunk continuously to the LQ fixed point.

the scale factor $a$. Defining

$$
\rho_{-}=\frac{3}{\kappa a^{2}}, \quad \rho_{+}=\rho_{c}+\frac{3}{\kappa a^{2}}, \quad \rho_{m}=\rho+\frac{\Lambda}{\kappa}
$$

equation (12) can be written in the form

$$
H^{2}=\frac{\kappa}{3 \rho_{c}}\left(\rho_{m}-\rho_{-}\right)\left(\rho_{+}-\rho_{m}\right)
$$

When $\rho_{m}=\rho_{-}(a)$ or $\rho_{+}(a)$, then $H=0$, so the system undergoes a bounce or starts a recollapsing phase, respectively. This is illustrated in Fig. 3. 


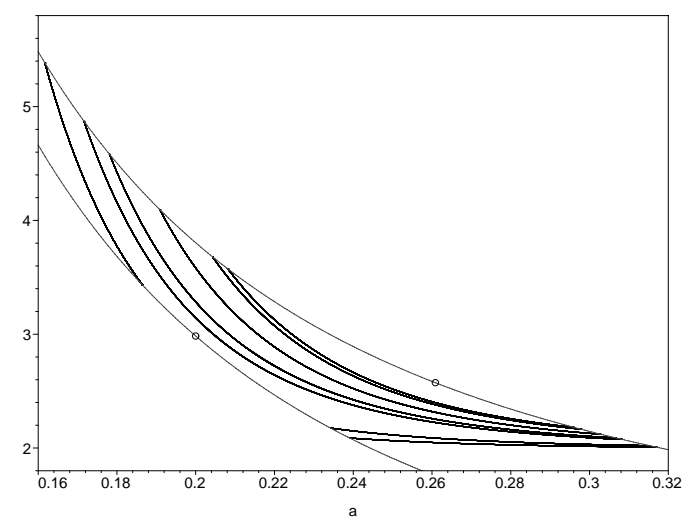

Figure 3. The upper and lower curves in grey are $\rho_{+}(a)$ and $\rho_{-}(a)$ respectively. Trajectories for several initial conditions are depicted in black. The GR fixed point is the box on he curve $\rho_{-}$, and the LQ point is the circle on the curve $\rho_{+}$.

We can integrate equation (14) for $\rho$ as a function of $a$, and substitute the expression for $a(\rho)$ into the modified Friedman equation (12). Using the dimensionless variables,

$$
x^{2}=\frac{3 \kappa \rho_{c}}{\Lambda^{2}} H^{2}, \quad y=\frac{\kappa \rho}{\Lambda}, \quad B=\frac{\kappa \rho_{c}}{\Lambda}
$$

this leads to

$$
x^{2}=\left[y+1-C y^{2 / 3(w+1)}\right]\left[B-y-1+C y^{2 / 3(w+1)}\right]
$$

where $C$ is a constant of integration. This expression allows us to get a better understanding of the particular case depicted in Fig. 2 and Fig. 3, where both the fixed points are present, by finding the separatrix. The separatrix is a curve (actually, a union of orbits) which marks the boundary of regions where the dynamical behaviour of the system is different. In this case the separatrix is the junction of the stable and unstable manifold with the GR Einstein static hyperbolic fixed point. First we solve equation (33) for $C$, then we substitute numerical values for the parameters $w=1$ and $B=.4121769562$ (using $M_{P}=1$ ), and we also substitute $x=0$ and $y=1 / 2$ for the GR fixed point. This produces a numerical value for $C$. Through this procedure, the equation of the separatrix is implicitly given by equation (33) with fixed values of the parameters $C, w$ and $B$. 
More insight can be acquired via the plot of the separatrix projected onto the $(x, y)$-plane, as shown in Fig. 4. The curves depicted are the separatrix, which wraps around the Friedman surface, and some other trajectories which are first integrals obtained for different values of the integration constants. The closed loops around the Friedman surface are outside the region marked by the separatrix; the other curve is not closed around the tube, and it can be shrunk continuously to the LQ fixed point.

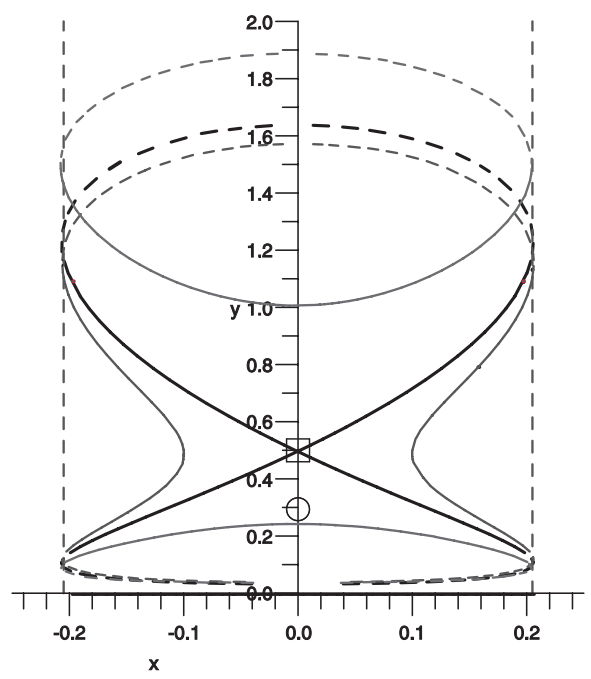

Figure 4. Projection of the phase space onto the $(x, y)$-plane for the case of Fig. 2. It represents the trajectories as seen from the bottom of the Friedman surface. Both the GR fixed point (a box) and the LQ fixed point (a circle) exist. The two vertical lines are the edges of the Friedman surface. The thick curve represents the two branches of the separatrix.

\section{Conclusions}

We have considered the application of dynamical systems theory to mathematical models from classical and semiclassical Cosmology. The ES universe in GR have been reviewed along with its stability properties in the case of a positive cosmological constant.

We have shown that LQC gravitational modifications to the Friedman equation lead to a new high-energy critical point for the Einstein static universe. This LQC Einstein static model is an unstable saddle (like the standard GR Einstein 
static solution) for sufficiently negative pressure and a sub-critical cosmological constant, i.e.

$$
-1<w<-\frac{1}{3}, \quad \Lambda<\kappa \rho_{c} .
$$

If $w$ is large enough and the cosmological constant is above the critical value, then the LQC Einstein static model is a centre:

$$
w>-\frac{1}{3}, \quad \Lambda>\kappa \rho_{c} .
$$

This neutrally stable behaviour is in strong contrast to the GR case, where the Einstein static model is unstable for all (positive) values of $\Lambda$.

This illustrates the general point that high-energy modifications to GR, which typically strongly modify the big bang singularity, also modify the dynamical nature of the non-singular Einstein static universe.

\section{Acknowledgements}

The author would like to thank the ICG, Portsmouth (UK), where part of this work was made, to Professor Gaetano Vilasi for continuous encouragement and stimulating discussion and to Ivaïlo M. Mladenov and his coworkers for warm hospitality in Bulgaria. The author was partially supported by PRIN 2006 and ASI.

\section{References}

[1] Arrowsmith C. and Place C., Dynamical Systems: Differential Equations, Maps and Chaotic Behaviour, Chapman \& Hall, N.Y., 1992.

[2] Barrow J., Ellis G., Maartens R. and Tsagas C., On the Stability of the Einstein Static Universe, Class. Quant. Grav. 20 (2003)] L155

See also, Losic B. and Unruh W., On the Initial Value Problem for Second Order Scalar Fluctuations in Einstein Static, Phys. Rev. D 71 (2005) 044011.

[3] Boehmer C., Hollenstein L. and F. S. N. Lobo, Stability of the Einstein Static Universe in $f(R)$ Gravity, Phys. Rev. D 76 (2007) 084005.

[4] Bojowald M., Absence of a Singularity in Loop Quantum Cosmology, Phys. Rev. Lett. 86 (2001) 5227-5230;

Ashtekar A. et al., Mathematical Structure of Loop Quantumcosmology, 
Adv. Theor. Math. Phys. 7 (2003) 233-268.

Ashtekar A., An Introduction to Loop Quantum Gravity Through Cosmology, Nuovo Cim. B 122 (2007) 135-155.

Bojowald M., Loop Quantum Cosmology, Living Rev. Rel. 11 (2008) 4.

[5] Borde A. and Vilenkin A., Eternal Inflation and the Initial Singularity, Phys. Rev. Lett 72 (1994) 3305-3308;

Borde A. and Vilenkin A., Violation of the Weak Energy Condition in Inflating Spacetimes, Phys. Rev. D 56 (1997) 717-723;

Guth A., Eternal Inflation, arXiv:astro-ph/0101507;

Guth A., Eternal Inflation and its Implications, J. Phys. A 40 (2007) 68116826.

[6] Clifton T. and Barrow J., The Existence of Goedel, Einstein and de Sitter Universes Phys. Rev. D 72 (2005) 123003.

[7] Eddington A., On the Instability of Einstein's Spherical World, Mon. Not. R. Astr. Soc. 90 (1930) 668-678.

[8] Eddington A., The Expansion of the Universe, Cambridge University Press, Cambridge, 1933;

Harrison E., Mon. Not. R. Astron. Soc. 137 (1967) 69;

Rebhan E., "Soft Bang" Instead of "Big Bang": Model of an Inflationary Universe without Singularities and with Eternal Physical Past Time, Astron. Astrophys. 353 (2000) 1-9.

[9] Ellis G. and Maartens R., The Emergent Universe: Inflationary Cosmology with no Singularity, Class. Quant. Grav. 21 (2004) 223-232;

Ellis G., Murugan J. and Tsagas C., The Emergent Universe: An Explicit Construction, Class. Quant. Grav. 21 (2004) 233-249;

Mukherjee S., Paul B., Dadhich N., Maharaj S. and Beesham A., Emergent Universe with Exotic Matter, Class. Quant. Grav. 23 (2006) 6927-6933.

[10] Gergely L. and Maartens R., Brane-world Generalizations of the Einstein Static Universe, Class. Quant. Grav. 19 (2002) 213-221.

[11] Hawking S. and Ellis G., The Large Scale Structure of Space-Time, Cambridge University Press, Cambridge, 1975.

[12] Mulryne D., Tavakol R., Lidsey J. and Ellis G., An Emergent Universe from a Loop, Phys. Rev. D 71 (2005) 123512.

[13] Parisi L., Bruni M., Maartens R. and Vandersloot K., The Einstein Static Universe in Loop Quantum Cosmology, Class. Quant. Grav. 24 (2007) 62436253. 
[14] Seahra S. and Boehmer C., Einstein Static Universes are Unstable in Generic $f(R)$ Models, arXiv:0901.0892 [gr-qc].

[15] Singh P., Transcending Big Bang in Loop Quantum Cosmology: Recent Advances, J. Phys. Conf. Ser. 140 (2008) 012005

[16] Spergel D. et al. [WMAP Collaboration], Wilkinson Microwave Anisotropy Probe (WMAP) Three Year Results: Implications for Cosmology, Astrophys. J. Suppl. 170 (2007) 377;

Springel V., Frenk C. and White S., The Large-scale Structure of the Universe, Nature 440 (2006) 1137-1144.

[17] Thiemann T., Introduction to Modern Canonical Quantum General Relativity, CUP, Cambridge, in press;

Rovelli C., Quantum Gravity, CUP, Cambridge, 2004;

Ashtekar A. and Lewandowski J., Background Independent Quantum Gravity: a Status Report, Class. Quant. Grav. 21 (2004) R53.

[18] Wainwright J., Ellis G., Dynamical Systems in Cosmology, Cambridge University Press, Cambridge, 1997.

[19] Wald R., General Relativity, Chicago University Press, Chicago, 1984.

Luca Parisi

Istituto Nazionale di Fisica Nucleare

Sezione di Napoli, GC di Salerno

Dipartimento di Fisica "E. R. Caianiello"

Universit'a di Salerno

Via S.Allende, I

84081 Baronissi (Salerno)

ITALY

E-mail address: parisi@sa.infn.it 